\title{
Clinical Study \\ Utility of Computed Tomographic Enteroclysis/Enterography for the Assessment of Mucosal Healing in Crohn's Disease
}

\author{
Shinichi Hashimoto, ${ }^{1}$ Kensaku Shimizu, ${ }^{2}$ Hiroaki Shibata, ${ }^{1}$ Satoko Kanayama, \\ Ryo Tanabe, ${ }^{3}$ Hideko Onoda, ${ }^{2}$ Naohumi Matsunaga, ${ }^{2}$ and Isao Sakaida ${ }^{1}$ \\ ${ }^{1}$ Department of Gastroenterology and Hepatology, Yamaguchi University Graduate School of Medicine, \\ 1-1-1 Minami-Kogushi, Ube, Yamaguchi 755-8505, Japan \\ ${ }^{2}$ Department of Radiology, Yamaguchi University Graduate School of Medicine, 1-1-1 Minami-Kogushi, \\ Ube, Yamaguchi 755-8505, Japan \\ ${ }^{3}$ Iryouhoujin-Seijinkai Hayashi Hospital, 751-4 Ogoori-Shimogou, Yamaguchi, Yamaguchi 754-0002, Japan \\ Correspondence should be addressed to Shinichi Hashimoto; has-333@yamaguchi-u.ac.jp
}

Received 23 January 2013; Accepted 7 April 2013

Academic Editor: Jan Bures

Copyright (C) 2013 Shinichi Hashimoto et al. This is an open access article distributed under the Creative Commons Attribution License, which permits unrestricted use, distribution, and reproduction in any medium, provided the original work is properly cited.

\begin{abstract}
Aim. When determining therapeutic strategy, it is important to diagnose small intestinal lesions in Crohn's disease (CD) precisely and to evaluate mucosal healing as well as clinical remission in CD. The purpose of this study was to compare findings from computed tomographic enteroclysis/enterography (CTE) with those from the mucosal surface and to determine whether the state of mucosal healing can be determined by CTE. Materials and Methods. Of the patients who underwent CTE for CD, 39 patients were examined whose mucosal findings could be confirmed by colonoscopy, capsule endoscopy, balloon endoscopy, or with the resected surgical specimens. Results. According to the CTE findings, patients were determined to be in the active CD group $(n=31)$ or inactive CD group $(n=8)$. The proportion of previous surgery, clinical remission, stenosis, and CDAI score all showed significant difference between groups. Mucosal findings showed an association with ulcer in $93.6 \%$ of active group patients but in only $12.5 \%$ of inactive group patients $(P<0.0001)$, whereas mucosal healing was found in $62.5 \%$ of inactive group patients but in only $3.2 \%$ of active group patients $(P<0.0001)$. Conclusion. CTE appeared to be a useful diagnostic method for assessment of mucosal healing in Crohn's disease.
\end{abstract}

\section{Introduction}

Because the small intestine is long and is located between the stomach and large intestine, small bowel examination is difficult. Now, however, with the advent of capsule endoscopy and balloon endoscopy, the ability to diagnose small bowel disease has progressed rapidly $[1,2]$.

Because it has been reported that more than $75 \%$ of patients with Crohn's disease have an active lesion in the small intestine, and one third of patients with Crohn's disease have an active lesion only in the small intestine [3], it is important in determining therapeutic strategy to diagnose lesions of Crohn's disease in the small intestine precisely. Furthermore, in recent years, biologics have become available for the treatment of Crohn's disease, and it is important to evaluate mucosal healing as well as clinical remission from Crohn's disease treatment at the appropriate time [4]. Presently, capsule endoscopy for Crohn's disease is performed in Japan, but there is the risk of retention of the capsule endoscope in cases of severe stenosis, and insertion of the balloon endoscope deeply into the small intestine is difficult in such cases.

Computed tomographic (CT) enteroclysis is one of the modalities for examination of the small intestine and is performed with the small intestine expanded by injection of a liquid contrast medium through a nasoduodenal tube [5]. In CT enterography, however, the patient ingests the liquid contrast medium orally without using a nasoduodenal tube [6]. The utility of CT enteroclysis/enterography (CTE) for Crohn's disease has been shown in numerous reports, but those reports have mainly focused on the diagnosis of stenosis or fistula $[7,8]$. There are no reports, to our knowledge, that compare in detail findings of CTE with those of the mucosal surface. 


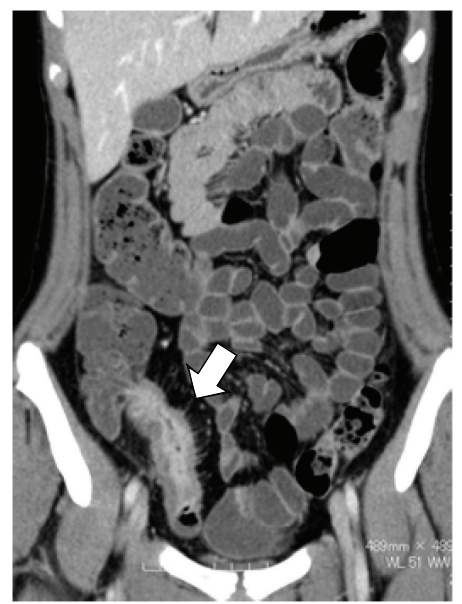

(a)

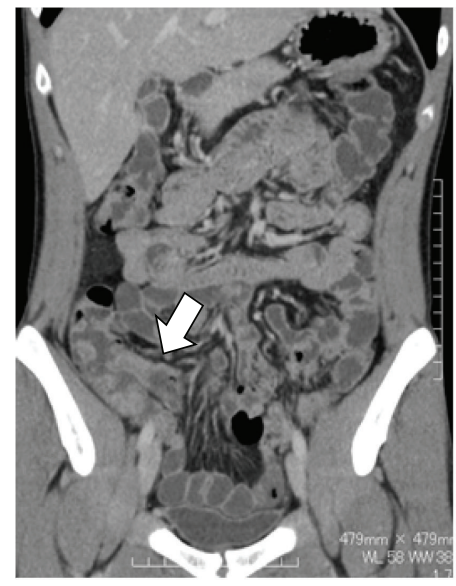

(c)

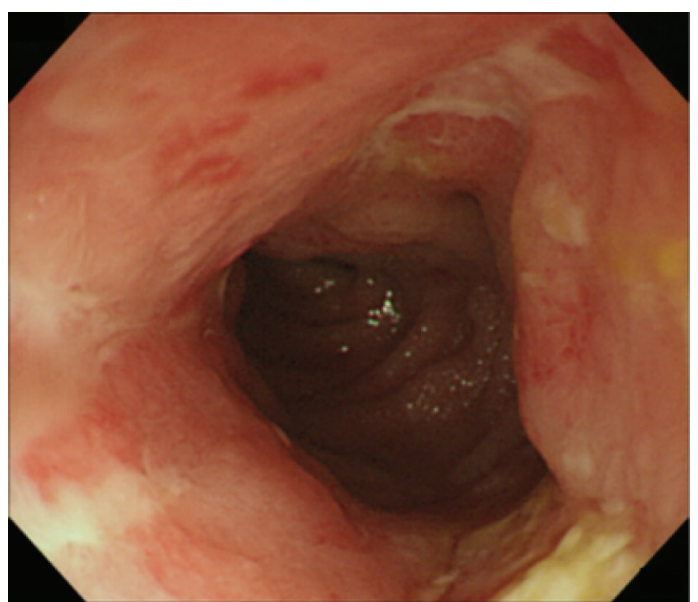

(b)

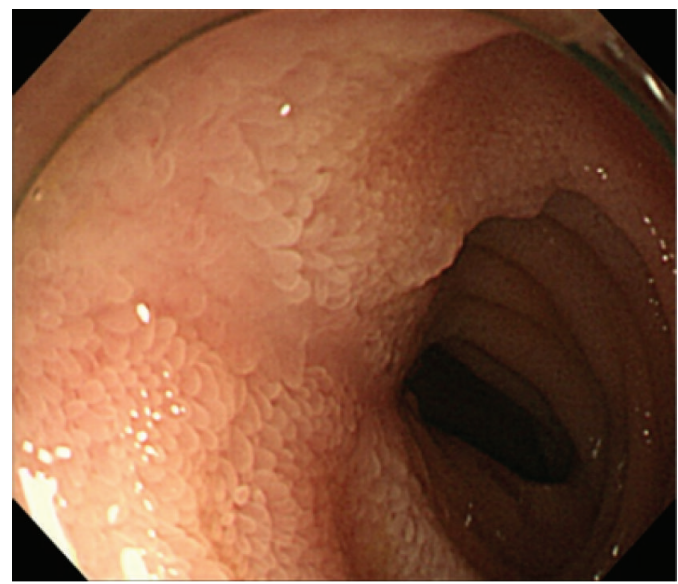

(d)

FIGURE 1: Findings from CT enteroclysis/enterography and endoscopic mucosal examination. (a) CT enteroclysis/enterography findings from a patient in the active group show small bowel wall thickness, wall enhancement, and presence of an increase in the concentration of fat tissue surrounding the intestine. (b) Mucosal findings corresponding to the same location as indicated by the arrow in (a) show edema and ulcer. (c) CT enteroclysis/enterography findings from a patient in the inactive group show only slight small bowel wall thickness. (d) Mucosal findings corresponding to the same location as indicated by the arrow in (c) show only an ulcer scar.

Therefore, the purpose of the present study was to compare the findings from CTE with those from examination of the mucosal surface and to determine whether the state of mucosal healing can be determined by CTE.

\section{Materials and Methods}

2.1. Patients. Of the patients who underwent CTE for Crohn's disease at our hospital between January 2009 and December 2012, the cases of 39 patients were examined whose mucosal findings could be confirmed by colonoscopy, capsule endoscopy, balloon endoscopy, or with the surgically resected specimens. Clinical activity was assessed with the Crohn's disease activity index (CDAI). Clinical remission was defined as a CDAI score of less than 150 .

2.2. CT Enteroclysis/Enterography. CT enteroclysis was first reported by Klöppel et al. in 1992 [9], and the procedure we used was as follows. First, a transnasal endoscope was inserted into the duodenum. A guide wire was then inserted into the jejunum through the forceps channel of the endoscope, and a $16 \mathrm{~F}$ balloon-tipped nasoduodenal tube was inserted into the duodenojejunal flexure along the guide wire after removing the transnasal endoscope. About 1200 $1800 \mathrm{~mL}$ of polyethylene glycol solution (PEG) at a temperature of about $37^{\circ} \mathrm{C}$ was then infused into the small intestine at a rate of $150 \mathrm{~mL} / \mathrm{min}$ with a power injector after inflating the balloon at the tip of the tube [10]. Immediately after infusing the PEG, the patient was transferred to the CT unit (SOMATOM Definition Dual Source CT; Siemens, Erlangen, Germany). After undergoing unenhanced CT, the patient received $100 \mathrm{~mL}$ of nonionic contrast medium by means of a power injector for the contrast-enhanced study. The contrast material was injected intravenously at a monophasic rate of injection of $3.0 \mathrm{~mL} / \mathrm{s}$. Three-phase scanning was begun at 40,70 , and $120 \mathrm{~s}$ after the start of the injection. The entire abdomen and pelvis were scanned using breath-hold acquisition during each phase with parameters of $0.6 \mathrm{~mm}$ 
collimation and a pitch of 1.2. Images were reconstructed at $2 \mathrm{~mm}$ intervals, and multiplanar views were created on an attached workstation.

With reference to the report by Huprich and Fletcher [6], CT enterography was performed with 1000-1800 mL of PEG that was divided into four doses and administered orally in 1h. The CT scanning method was the same as that for CT enteroclysis.

Because PEG is rapidly infused by nasoduodenal tube, small intestine is thought to be distended more uniformly in CT enteroclysis than in CT enterography. Meanwhile, mechanical rapid infusion could increase the risk of ileus or perforation in the case with severe stenosis. For this reason, CT enterography was chosen for the patients with suspected or established severe stenosis. We performed CT enteroclysis on 20 patients and CT enterography on 19 patients. Although an ileus occurred in one patient after CTE, it soon improved with conservative treatment. The CTE protocol was approved by the Ethics Committee of the Yamaguchi University Graduate School of Medicine.

2.3. Evaluation of CTE Findings. All patients were divided into either the active group or inactive group according to CTE findings. Active group patients comprised those in whom wall thickening was found with early contrast enhancement and an increase in the concentration of fat tissue surrounding the intestine was present at one or more segment. Inactive group patients comprised those in whom there was no significant increase in early contrast enhancement and no concentration of fat tissue surrounding the intestine, even if CTE showed wall thickening (Figure 1).

2.4. Evaluation of Mucosal Findings. Mucosal findings were evaluated by colonoscopy, capsule endoscopy, balloon endoscopy, or examination of the resected specimen. Colonoscopy was performed with a CF-H260AZI, PCF-Q260AZI, PCFQ260J, or PCF-PQ260I colonoscope (Olympus Corporation, Tokyo, Japan). The PillCam SB capsule endoscopy system (Given Imaging, Yokneam, Israel) was used for capsule endoscopy, and image interpretation was carried out on a Rapid Reader (Version 6.5; Given Imaging). Balloon endoscopy was performed with an EN-450T5/W (Fujifilm Corporation, Tokyo, Japan) or SIF-Q260 (Olympus Corporation) endoscope. According to findings of the mucosal surface, mucosal healing was defined as the absence of erosion or ulcer.

2.5. Statistical Analysis. The $t$-test and chi-square test were used for univariate analysis. Differences were considered statistically significant at a value of $P<0.05$. Statistical analysis was performed with StatView 5 (Abacus Concepts, Berkeley, CA, USA).

\section{Results}

According to the CTE findings, the active group comprised 31 patients, and the inactive group comprised 8 patients. We compared the clinical background of each group and found no significant differences in sex ratio and age. The
TABLE 1: Characteristics of the two study groups (univariate analysis).

\begin{tabular}{lccc}
\hline & $\begin{array}{c}\text { Active } \\
(n=31)\end{array}$ & $\begin{array}{c}\text { Inactive } \\
(n=8)\end{array}$ & $P$ value \\
\hline Sex, male (\%) & 64.5 & 37.5 & 0.1660 \\
Age (years) & $36.5 \pm 17.4$ & $37.8 \pm 15.3$ & 0.8485 \\
Previous surgery (\%) & 35.5 & 0 & $<0.05$ \\
Biologics use (\%) & 22.6 & 37.5 & 0.3889 \\
CDAI & $196.1 \pm 81.2$ & $79.1 \pm 59.6$ & $<0.001$ \\
Clinical remission (\%) & 29.0 & 87.5 & $<0.01$ \\
Stenosis (\%) & 80.6 & 25.0 & $<0.01$ \\
Fistula (\%) & 12.9 & 0 & 0.2835 \\
Abscess (\%) & 3.2 & 0 & 0.6126 \\
\hline
\end{tabular}

CDAI: Crohn's disease activity index.

TABLE 2: Mucosal findings of the two study groups (univariate analysis).

\begin{tabular}{lccc}
\hline Mucosal findings & Active $(n=31)$ & Inactive $(n=8)$ & $P$ value \\
\hline Mucosal healing & $1(3.2 \%)$ & $5(62.5 \%)$ & $<0.0001$ \\
Erosion $(\%)$ & $1(3.2 \%)$ & $2(25.0 \%)$ & $<0.05$ \\
Ulcer $(\%)$ & $29(93.6 \%)$ & $1(12.5 \%)$ & $<0.0001$ \\
\hline
\end{tabular}

proportion of previous surgery, clinical remission, stenosis, and CDAI scorewas significantly different between the two groups. Although there was no significant difference between the two groups in the proportion of fistulae and abscesses, these were observed only in the active group (Table 1).

With respect to the mucosal findings, an association was found with ulcer in $93.6 \%$ of patients in the active group but in only $12.5 \%$ of patients in the inactive group. Mucosal healing was found in $62.5 \%$ of patients in the inactive group and in only $3.2 \%$ of patients in the active group (Table 2 ).

In a patient who underwent CTE before and after the introduction of biologics, the second CTE was undergone at 84 days after the first CTE. Despite a slight change in the CDAI score from 112 to 88 , enhancement of the contrast effect and marked edema of the ileo cecum were improved at the second CTE compared with that observed at the first CTE, and corresponding changes were also seen in the endoscopic findings (Figure 2).

\section{Discussion}

The present study revealed a strong correlation between CTE findings and mucosal surface findings in patients with Crohn's disease. Because ulcers were found in $93.6 \%$ of patients in the active group, CTE findings in active group suggested that ulcers frequently exist in the mucosa. Because it is difficult to diagnose drug-related ulcers in the small intestine (e.g., from NSAIDs) by CTE (data not shown), the reason the ulcers were detected by CTE in Crohn's disease is that this disease is characterized by transmural inflammation. In the inactive group, $62.5 \%$ of patients were classified as having mucosal healing, and the ulcer rate in the inactive group was 


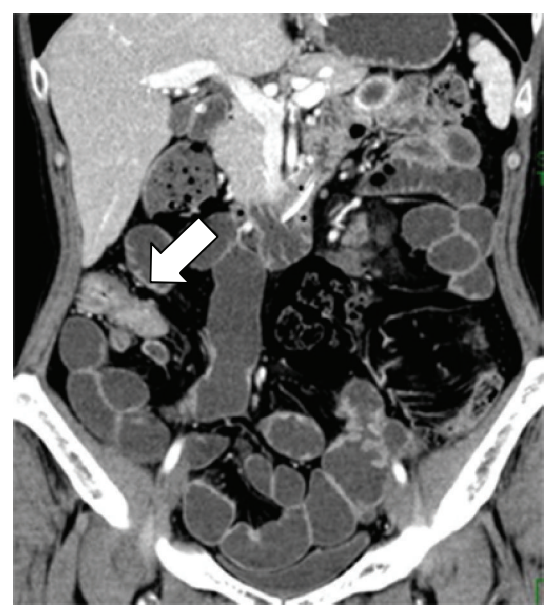

(a)

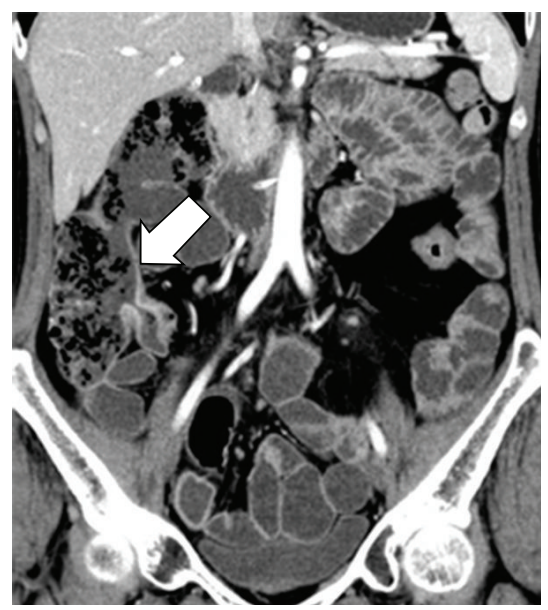

(c)

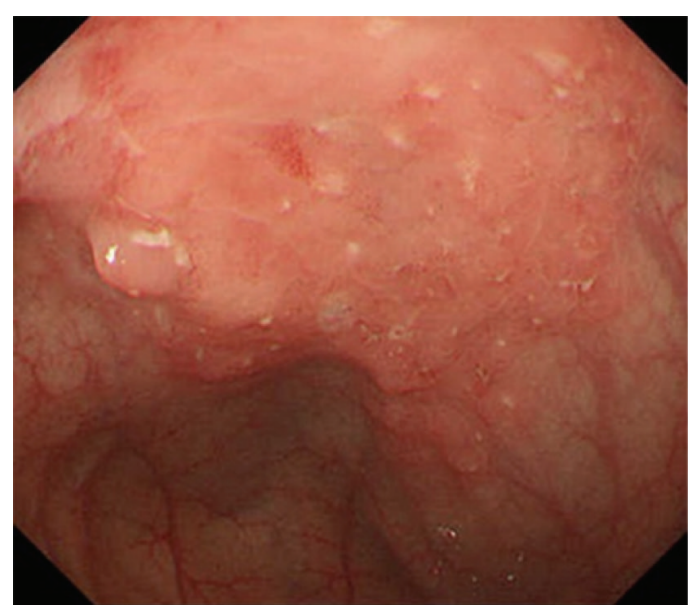

(b)

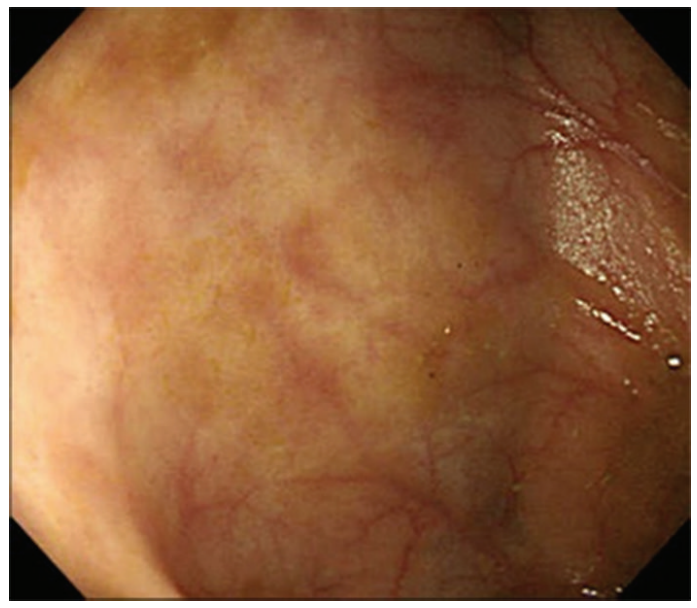

(d)

FIgURE 2: Findings from CT enteroclysis and endoscopic mucosal examination before and after the introduction of biologics. (a) CT enteroclysis before the introduction of biologics. (b) Mucosal findings corresponding to the same location as indicated by the arrow in (a) show edema. (c) CT enteroclysis at 84 days after the first CT enteroclysis showing improvement of the inflammation. (d) Mucosal findings corresponding to the same location as indicated by the arrow in (c) show no edema or ulcer.

significantly lower than that in the active group. However, two patients with erosion and one patient with ulcer were observed in the inactive group. The reason why CTE could not detect these lesions was thought to be that inflammation of the mucosa surrounding the lesion was slight.

With regard to clinical background factors, the percentage of clinical remission and CDAI scores were significantly different between the active and inactive groups. CTE findings were found to be associated with clinical activity. However, even though clinical remission was determined in $29.0 \%$ of the active group patients and clinical symptoms were not recognized, active inflammation could be observed in the intestine of these patients. Thus, even if patients are experiencing clinical remission, they should regularly undergo evaluation of the mucosal surface to prevent the progression of complications.

There are several ways to observe the small intestine: colonoscopy, capsule endoscopy, and balloon endoscopy. In colonoscopy, it is difficult to insert the colonoscope into the ileum in about $15 \%$ of cases [11]. Furthermore, inflammatory lesions might exist outside the reachable range of the colonoscope. Because one of the major limitations of capsule endoscopy is capsule retention, this procedure cannot be undergone by patients with severe stenosis or with failed and uncertain passage of a patency capsule [12]. It is difficult to observe the entire small intestine by balloon endoscopy, and the patient requires conscious sedation during the procedure. Thus, it is thought that CTE will become the method of choice to assess Crohn's disease activity in the small intestine, especially for the case with severe stenosis.

In addition to assessment of inflammation in the small intestine, CTE has been reported to be useful for diagnosis of stenosis and fistula in Crohn's disease. Vogel et al. reported that CT enterography showed a high detection capability for stenosis and fistula in patients who underwent surgery within 3 months after CT enterography [7]. Wold et al. reported that CT enterography protocol had similar accuracy of active Crohn's disease in comparison with CT enteroclysis [13]. In 
the present study, CTE not only provided information on the mucosa, but it also detected stenoses, fistulae, and abscess in the abdominal cavity.

One of the problems with CTE for Crohn's disease is the amount of radiation exposure received by the patient. Cumulative exposure to exceeding $75 \mathrm{mSv}$ radiation has previously been estimated to increase mortality due to all cancers by $7.3 \%$ [14]. Because of the young age of onset of Crohn's disease, CTE for this disease must be performed at an appropriate time. To help resolve this problem, the utility of MR enterography was reported as an alternative to CTE in the evaluation of young patients with Crohn's disease [15]. However, MR enterography has not become widespread, especially in Japan, due to its lack of availability and limited expertise with the procedure [16]. The usefulness of CT dose reduction with CTE has been reported $[16,17]$. By means of reductionof radiation doses and image noise reduction methods, Lee et al. reported that the mean effective doses were $4.7 \mathrm{mSv}$ and $2.4 \mathrm{mSv}$ for standard-dose CTE and lowdose CTE examinations, respectively [17]. To enhance the usefulness of low-dose CTE, it is necessary to study the relation between CTE findings and the mucosal surface in more detail and to create a classification system of CTE findings to use when determining mucosal healing with CTE. After this, effective timing of CTE in the therapeutic strategy for Crohn's disease will become apparent.

\section{Conclusions}

CTE appeared to be a useful diagnostic method for the assessment of mucosal healing in Crohn's disease.

\section{Acknowledgment}

The authors express their gratitude to Dr. Shingo Higaki, Chief of the Department of Gastroenterology and Hepatology, St. Hill Hospital, for his guidance and useful discussions.

\section{References}

[1] G. Iddan, G. Meron, A. Glukhovsky, and P. Swain, "Wireless capsule endoscopy," Nature, vol. 405, no. 6785, pp. 417-418, 2000.

[2] H. Yamamoto, H. Kita, K. Sunada et al., "Clinical outcomes of double-balloon endoscopy for the diagnosis and treatment of small-intestinal diseases," Clinical Gastroenterology and Hepatology, vol. 2, no. 11, pp. 1010-1016, 2004.

[3] P. F. Engstrom and E. B. Goosenberg, Diagnosis and Management of Bowel Diseases, Professional Communication Publishers, 1999.

[4] F. Baert, L. Moortgat, G. Van Assche et al., "Mucosal healing predicts sustained clinical remission in patients with early-stage Crohn's disease," Gastroenterology, vol. 138, no. 2, pp. 463-468, 2010.

[5] D. D. T. Maglinte, K. Sandrasegaran, J. C. Lappas, and M. Chiorean, "CT enteroclysis," Radiology, vol. 245, no. 3, pp. 661-671, 2007.

[6] J. E. Huprich and J. G. Fletcher, "CT enterography: principles, technique and utility in Crohn's disease," European Journal of Radiology, vol. 69, no. 3, pp. 393-397, 2009.
[7] J. Vogel, A. da Luz Moreira, M. Baker et al., "CT enterography for Crohn's disease: accurate preoperative diagnostic imaging," Diseases of the Colon and Rectum, vol. 50, no. 11, pp. 1761-1769, 2007.

[8] M. D. Kohli and D. D. T. Maglinte, "CT enteroclysis in small bowel Crohn's disease," European Journal of Radiology, vol. 69, no. 3, pp. 398-403, 2009.

[9] R. Klöppel, J. Thiele, and J. Bosse, "CT, Sellink method," RöFo, vol. 156, no. 3, pp. 291-292, 1992 (German).

[10] Y. B. Liu, C. H. Liang, Z. L. Zhang et al., "Crohn disease of small bowel: multidetector row $\mathrm{CT}$ with $\mathrm{CT}$ enteroclysis, dynamic contrast enhancement, CT angiography, and 3D imaging," Abdominal Imaging, vol. 31, no. 6, pp. 668-674, 2006.

[11] S. Cherian and P. Singh, "Is routine ileoscopy useful? An observational study of procedure times, diagnostic yield, and learning curve," American Journal of Gastroenterology, vol. 99, no. 12, pp. 2324-2329, 2004.

[12] F. T. Fork, N. Karlsson, S. Kadhem, and B. Ohlsson, "Small bowel enteroclysis with magnetic resonance imaging and computed tomography in patients with failed and uncertain passage of a patency capsule," BMC Medical Imaging, vol. 12, p. 3, 2012.

[13] P. B. Wold, J. G. Fletcher, C. D. Johnson, and W. J. Sandborn, "Assessment of small bowel Crohn disease: noninvasive peroral CT enterography compared with other imaging methods and endoscopy-feasibility study," Radiology, vol. 229, no. 1, pp. 275281, 2003.

[14] M. Vrijheid, E. Cardis, M. Blettner et al., "The 15-country collaborative study of cancer risk among radiation workers in the nuclear industry: design, epidemiological methods and descriptive results," Radiation Research, vol. 167, no. 4, pp. 361-379, 2007.

[15] S. L. Seung, Y. K. Ah, S. K. Yang et al., "Crohn disease of the small bowel: comparison of CT enterography, MR enterography, and small-bowel follow-through as diagnostic techniques," Radiology, vol. 251, no. 3, pp. 751-761, 2009.

[16] A. R. Kambadakone, N. A. Chaudhary, G. S. Desai, D. D. Nguyen, N. M. Kulkarni, and D. V. Sahani, "Low-dose MDCT and CT enterography of patients with crohn disease: feasibility of adaptive statistical iterative reconstruction," American Journal of Roentgenology, vol. 196, no. 6, pp. W743-W752, 2011.

[17] S. J. Lee, S. H. Park, A. Y. Kim et al., "A prospective comparison of standard-dose CT enterography and $50 \%$ reduced-dose CT enterography with and without noise reduction for evaluating Crohn disease," American Journal of Roentgenology, vol. 197, no. 1, pp. 50-57, 2011. 


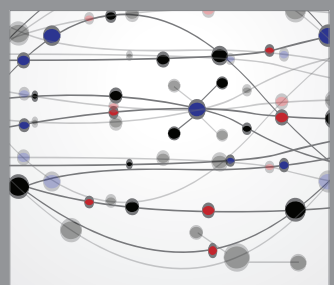

The Scientific World Journal
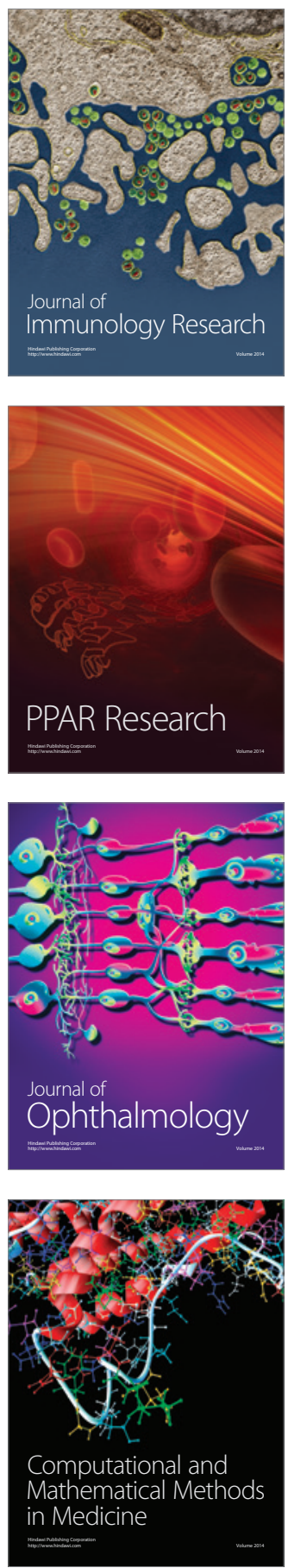

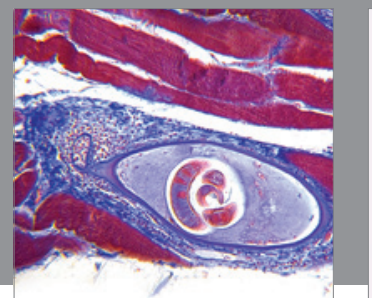

Gastroenterology

Research and Practice
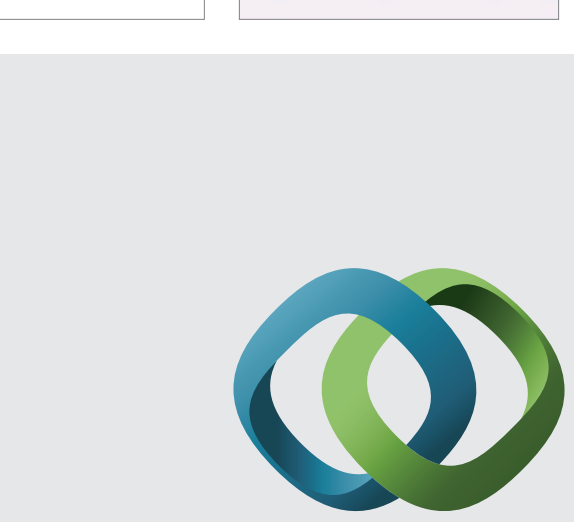

\section{Hindawi}

Submit your manuscripts at

http://www.hindawi.com
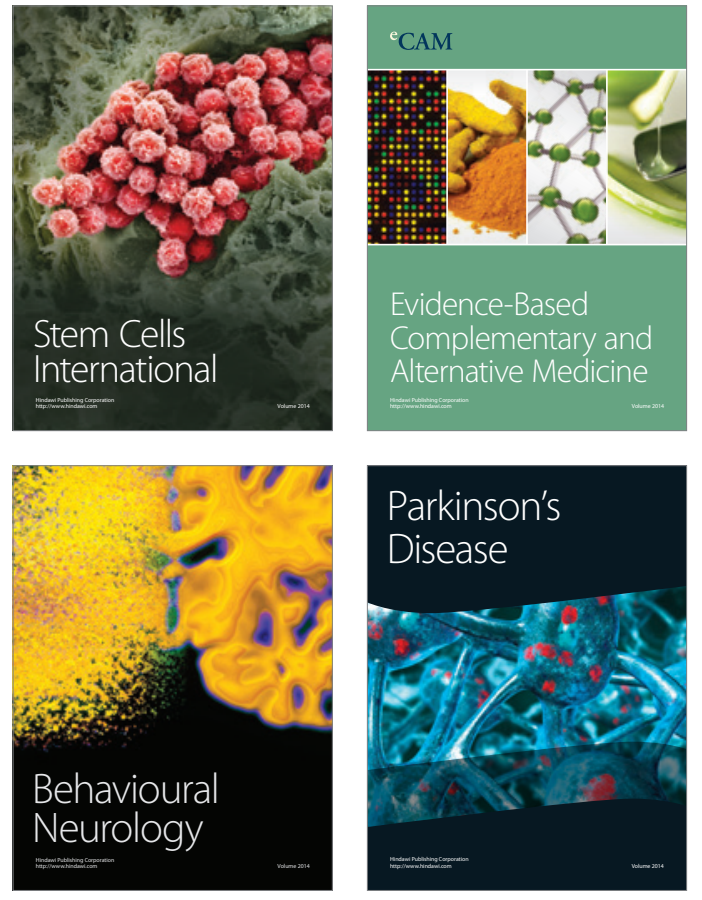
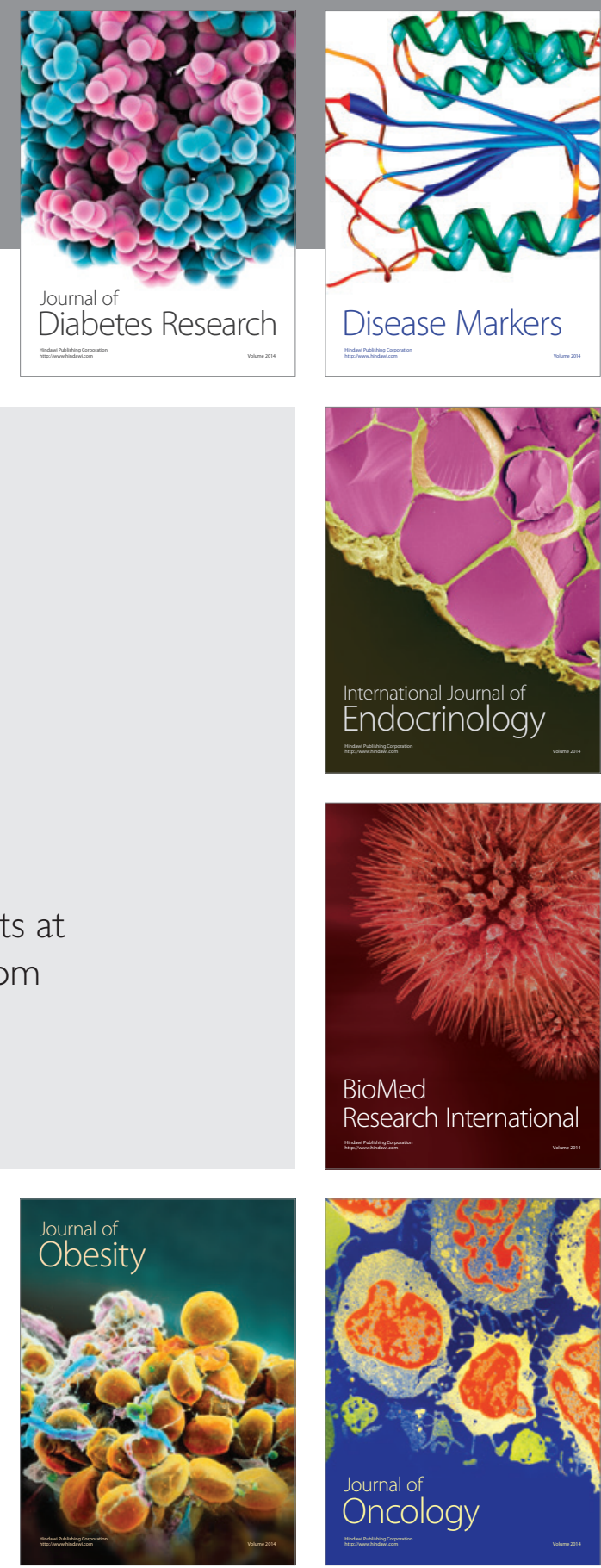

Disease Markers
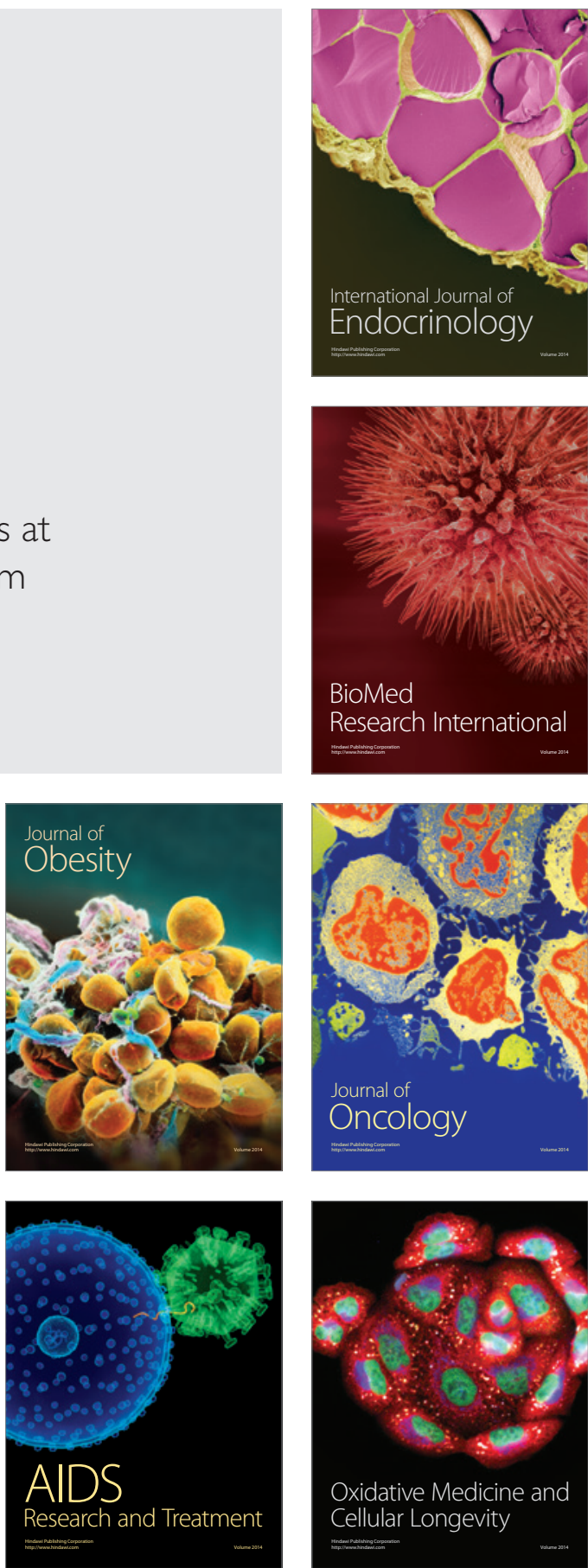